\title{
Optically induced oxygen desorption from graphene measured using femtosecond two-pulse correlation
}

\author{
S. M. Hornett, M. Heath, D. W. Horsell, and E. Hendry \\ School of Physics, University of Exeter, Exeter EX4 4QL, United Kingdom \\ (Received 8 March 2014; revised manuscript received 4 July 2014; published 4 August 2014)
}

\begin{abstract}
Recently, there has been a great deal of interest in the effect of atmospheric gases on the properties of graphene. We investigate the electrical and optical response of graphene-based field effect transistors that have been exposed to high purity oxygen gas using a combination of ultrafast two-pulse correlation (to give high temporal resolution) and low-frequency transport measurements (to monitor the photoinduced changes in the Fermi level). By measuring the Fermi level shifts, we are only sensitive to the oxygen atoms that interact directly with the surface. We compare our results to predictions of the empirical friction model for molecular desorption. We show the time scale of the relaxation associated with oxygen desorption to be $\sim 100 \mathrm{fs}$, suggesting the desorption proceeds through hot electron generation in the graphene rather than heating of the lattice through hot phonon generation.
\end{abstract}

DOI: 10.1103/PhysRevB.90.081401

PACS number(s): 78.67.Wj, 78.47.J-

Ultrafast laser measurements on graphene have afforded great insight into quasiparticle relaxation mechanisms and lifetimes in this material [1-4]. For example, it is now known that, after ultrafast photoexcitation of the electrons, energy relaxation occurs through mechanisms with markedly different time scales: first through energy redistribution by electron-electron scattering ( $\sim 10 \mathrm{fs})$, then thermalization with optical phonons ( $\sim 100 \mathrm{fs})$, and finally, anharmonic decay of optical phonons and/or coupling to substrate phonons $(\sim 1 \mathrm{ps})$ [2-4]. More recently, ultrafast laser measurements have been used to probe the time scales of charge transfer to graphene from photoexcited adsorbed molecules [5]. However, experimentally the exact time scales and mechanisms associated with the adsorption and desorption of molecular species are not well known [6].

Molecules that interact with graphene can significantly influence its electrical, optical, and mechanical properties [7-9]. This has led to graphene being used as the active element in a range of sensing applications [10]. Studies of changes to these properties have led to an understanding of the orientation of adsorbed molecular species [11], chemical activity mediated by the surface [12], and the type of electron scattering caused [13]. Adsorbed gases have also been shown to affect the $\mathrm{THz}$ conductivity of graphene [14]. Of particular interest is the interaction of graphene with molecular oxygen. Oxygen molecules can adsorb onto the surface of a pristine graphene sheet resulting in fractional charge transfer from the graphene to the oxygen. Recent studies have shown the importance of the substrate and environmental conditions to this charge transfer $[15,16]$. However, the nature of the interaction with oxygen has yet to be fully explored, and it is unclear exactly how and where the oxygen molecules bind to graphene and how efficiently molecules can dissociate at these sites.

Here, we investigate the mechanisms, energies, and time scales relevant for the molecular adsorption of oxygen molecules on graphene. To do this, we have designed an experiment which permits high electrical sensitivity to adsorbed molecular species coupled with the time resolution offered by ultrafast laser pulses. By monitoring small changes in electrical conductivity due to adsorbed molecular oxygen as a function of the time delay between pairs of correlated laser pulses, we show that the energy relaxation time scale associated with desorption of oxygen is very fast: $\sim 100$ fs. This indicates that a nonthermal, hot-electron mechanism is the dominant desorption pathway. Such rapid energy dissipation by hot electrons may explain the relative inefficiency of photochemical processes in graphene [17].

Graphene flakes were mechanically exfoliated onto degenerately doped silicon substrates covered by a 300-nm-thick $\mathrm{SiO}_{2}$ layer. Flakes were identified as single layers by optical contrast measurements and Raman spectroscopy. A voltage, $V_{g}$, applied between the doped silicon and graphene was used to control the carrier density in the graphene. Multiple electrical $\mathrm{Cr} / \mathrm{Au}$ contacts $(5 / 50 \mathrm{~nm})$ were made to the flakes to allow measurement of the differential resistance using a lowfrequency $(3 \mathrm{kHz})$ lock-in technique with an applied ac current of $6 \mu \mathrm{A}$. The samples were held in a high vacuum chamber (nominally $\sim 10^{-7}$ mbar at room temperature). Oxygen gas (99.5\% purity) was introduced into the chamber at atmospheric pressure to dose the graphene sample, before the chamber was again pumped down to high vacuum.

Adsorbed molecules on graphene can cause a shift in the Fermi level. This manifests itself as a shift of the resistance maximum at $V_{g}=V_{D}$ (corresponding to the Dirac point). This shift, $\Delta V_{D}$, can be used to determine the level of doping in the system. However, a simple, nongated measurement of the graphene resistance cannot distinguish mobility changes from shifts in the Fermi level. We therefore apply a sinusoidally varying voltage $(+/-30 \mathrm{~V}$ at $3 \mathrm{~Hz})$ to the gate, allowing the capture of a full $R\left(V_{g}\right)$ curve every $\approx 0.2 \mathrm{~s}$ (a rate and range that is limited by charging processes in the silicon). By tracking $V_{D}$ in real time, one can monitor changes in the charge transfer processes occurring between adsorbed molecules and the graphene layer, i.e., one can monitor changes in the binding of molecular adsorbates.

It is important to note that by using the Fermi level position of the graphene flake as a sensor we are only sensitive to molecules directly interacting with the graphene. This allows the measurement to be performed in a high vacuum chamber at room temperature rather than the ultrahigh vacuum needed for 

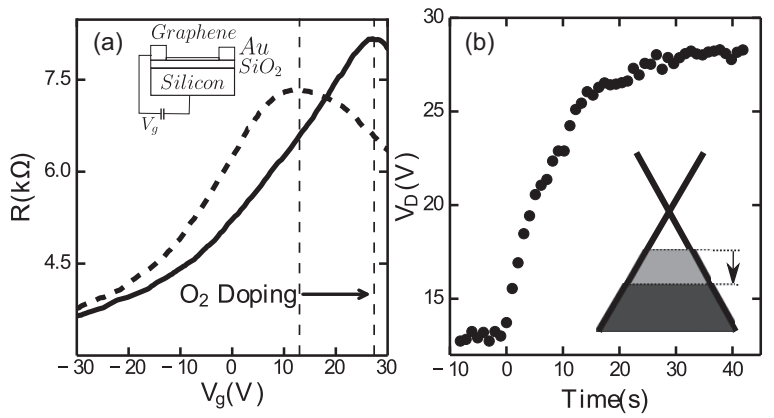

FIG. 1. (a) Resistance versus gate voltage before and after oxygen exposure (dashed and solid lines, respectively). The positions of the Dirac points are indicated by the dotted lines. The inset shows the sample layout. (b) The Dirac point position as a function of time after oxygen exposure. The oxygen was admitted to the chamber at $t=0 \mathrm{~s}$. The inset shows the band structure of graphene with the corresponding change in the Fermi level.

mass spectrometry techniques [18]. In order to photoexcite the samples, the vacuum chamber had optical access for $800 \mathrm{~nm}$, $100 \mathrm{fs}$ laser pulses with a $1 \mathrm{kHz}$ repetition rate. A variable mechanical shutter allowed control over sample exposure to laser pulses. In our experiments, effects due to steady state heating of the sample can be neglected, as we observed no dependence on the continuous exposure time. The pulse fluence of the laser was nominally $47 \mathrm{~J} \mathrm{~m}^{-2}$. The spot size of the beam is considerably larger than the average size of the graphene flakes: $4 \mathrm{~mm}$ and $\sim 10 \mu \mathrm{m}$, respectively.

Figure 1(a) shows the effect of adding oxygen at 1 atm to the chamber. This results in an increase in the peak resistance of the sample shown, though this effect varies from sample to sample. All samples tested, meanwhile, displayed an increase in the value of $V_{D}$ on exposure to oxygen. The shift of $V_{D}$ arises due to a fractional charge transfer from the graphene to the oxygen [19]. This shift eventually saturates to a value that is repeatable over multiple pumping-doping cycles as saturation adsorbate coverage is obtained. A control experiment with nitrogen was observed to have significantly smaller $\Delta V_{D}$. The maximum shift of $\sim 16 \mathrm{~V}$ for doping with oxygen [Fig. 1(b)] is equivalent to a change in carrier density of $11 \times 10^{11} \mathrm{~cm}^{-2}$ (calculated from net $=\epsilon_{0} \epsilon_{r} \Delta V_{D}$ where $\epsilon_{r}$ and $t$ are the permittivity and thickness of the $\mathrm{SiO}_{2}$ layer, respectively). Density functional theory (DFT) calculations for oxygen adsorption on graphene predict transfer of $\sim$ 0.01-0.02 electrons per molecule from graphene [19] with binding energies $<60 \mathrm{meV}$, although this depends on the density of states in the graphene [11]. For the saturation voltage observed in experiment, this would correspond to around $\sim 2 \%$ coverage of the graphene surface. However, we observe the binding of oxygen to graphene to be stable at room temperature $(\sim 26 \mathrm{meV})$ and under high vacuum. Indeed, it has previously been noted [11] that temperatures well above room temperature are needed to remove oxygen. This indicates binding energies are significantly greater than thermal energies at room temperature. This observation raises interesting questions about the nature of the binding, and suggests binding at defect sites and/or substrate interactions might play a role.
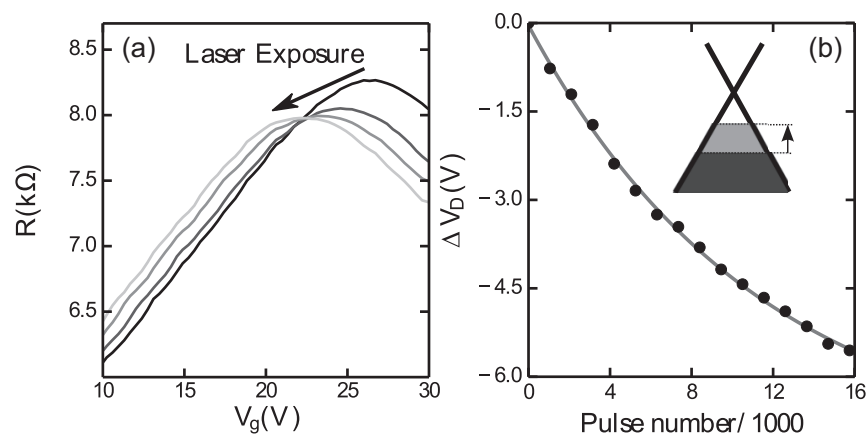

FIG. 2. (a) The evolution of the $R\left(V_{g}\right)$ curve with increasing laser exposure (black to gray). (b) The extracted Dirac point shift as a function of pulse number. This shows a reversal in sign of the doping behavior from the oxygen doping case. The data are fitted with an exponential decay (solid line).

In order to obtain information about the time scales and energies associated with desorption of oxygen, we expose the graphene to ultrafast laser pulses at near normal incidence under high vacuum. The gate voltage is set to zero during laser exposure to exclude any effect of surface charging on the desorption process [11]. Electromagnetic modeling [20] of the multilayer structure of the sample suggests that $\sim 1 \%$ of the incident fluence is absorbed by the graphene flake, corresponding to an absorbed energy density of $0.47 \mathrm{~J} \mathrm{~m}^{-2}$. This absorbed fluence is well below the damage threshold for graphene and significantly smaller than in some reported ultrafast measurements [21,22]. Also, we observe no defect peak in the Raman spectrum after several hours of exposure [21]. The dominant effect we observe on exposing the graphene to an increasing number of laser pulses is a shift of $V_{D}$ towards zero [Fig. 2(a)]. These photoinduced changes are stable in vacuum at room temperature, but almost completely reversible on redoping with oxygen, indicating that oxygen is being removed from the surface by photoexcitation. After several cycles of exposure and redoping we observe a slightly reduced desorption rate, which could be the result of stronger binding of the oxygen to photoinduced defects in graphene. We see no evidence for photodissociation of oxygen, which would result in a shift to higher $V_{D}$ due to increased binding and corresponding electron transfer.

Figure 2(b) shows the evolution of $V_{D}$ with increasing laser exposure. Each data point consists of nine averaged $R\left(V_{g}\right)$ measurements between which the sample is exposed to a $1 \mathrm{~s}$ train of pulses at $1 \mathrm{kHz}$. (Single pulse induced changes are inherently noisy due to pulse to pulse variations in the intensity profile of the beam, an effect which is diminished by using long pulse trains in the experiment). The Fermi level shift associated with excitation is then extracted by fitting a peaked function to the $R\left(V_{g}\right)$ curves. It is clear from the figure that each additional pulse train removes less oxygen from the surface. The line through the data points in Fig. 2(b) is an exponential fit. Such exponential behavior is characteristic of first order desorption kinetics, i.e., the rate of desorption is proportional to the density of adsorbed oxygen and characterized by a single rate constant. (We realize that there is likely more than one adsorption site at such an inhomogeneous interface, characterized by several different binding energies, but such 


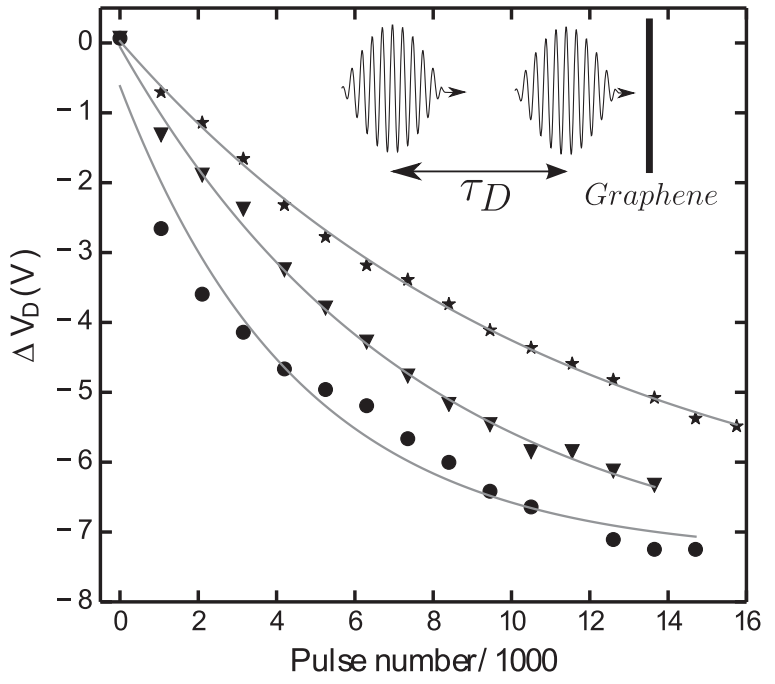

FIG. 3. Change in Dirac point position as a function of pulse number for three different delays between pairs of pulses. Circles are at overlap, triangles are $400 \mathrm{fs}$, and stars are at $800 \mathrm{fs}$. Solid lines correspond to an exponential fit to the data.

multiexponent behavior is not apparent above the noise of the experiment.) After several hours of exposure we observe that $V_{D}$ returns to a slightly higher voltage than observed before oxygen exposure, an effect we attribute to very strongly bound species (or even dissociated species) that are not removed at the power levels used.

To gain insight into the nature of the oxygen binding, we have carried out a two-pulse experiment. Such two-pulse correlation measurements have been applied to study the time scales and mechanisms of chemical processes at metallic interfaces [18] and in graphene to investigate the ultrafast photoluminescence [23]. The pulses are divided into two trains of equal magnitude and both converge at the graphene sample with a small $\left(\approx 5^{\circ}\right)$ separation in angle. One of the trains is sent through a delay line which introduces a delay $\tau_{d}$ between the pairs of laser pulses. Since the desorption process is nonlinear in nature (power dependence measurements suggest a power law dependence of order $\approx 4 \pm 0.8$ ), we can investigate the relevant time scales of the process by determining how desorption rates depend on $\tau_{d}$. In Fig. 3 we show the change in the Fermi level position as a function of exposure to laser pulse pairs of different delays between the two pulses. The greatest desorption rate is observed when the pulse pairs are overlapped in time (at $\tau_{d}=0$ ), and the effect per pulse diminishes very quickly as the correlated pulses are displaced in time. This effect is not due to coherent interference between the laser pulses when overlapped in time: We minimize any coherent artifacts by using orthogonal polarizations for the two pulses. We characterize the decays in Fig. 3 with an exponent (solid lines) and plot the decay rate extracted in Fig. 4(a) for multiple delay times.

It is known from optical pump-probe measurements that energy relaxation in graphene occurs via a three-stage process $[2,3,23,24]$ : Electron-electron interactions lead to a hot Boltzmann distribution of carriers within a few femtoseconds following photoexcitation [25]. Within $\sim 100$ fs following

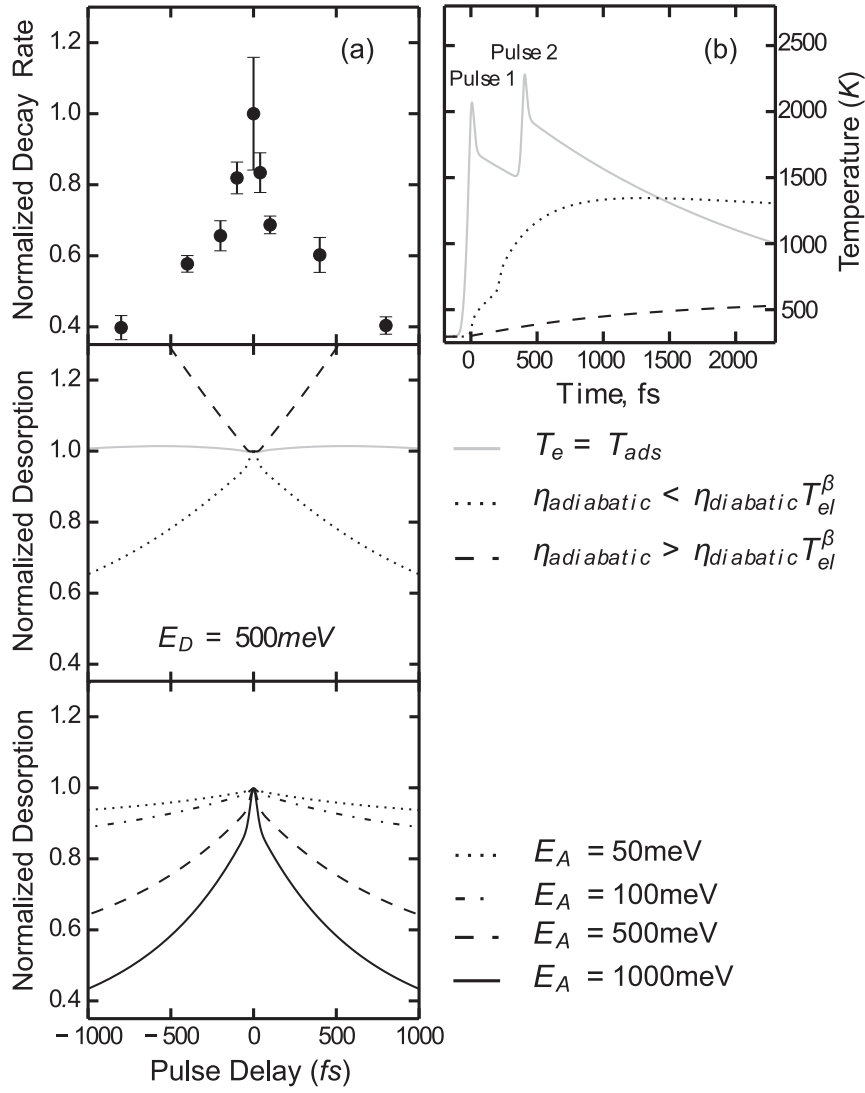

FIG. 4. (a) Top panel: Extracted decay rates from exponential fits to the data in Fig. 3. Middle panel: Integrated desorption rates predicted by the friction model for different values of $\eta_{\text {diabatic }}$ which give rise to the temperatures in (b). The dashed line shows the case corresponding to $\eta_{\text {adiabatic }}>\eta_{\text {diabatic }} T_{\text {el }}^{\beta}$, while the dotted line corresponds to $\eta_{\text {adiabatic }}<\eta_{\text {diabatic }} T_{\mathrm{el}}^{\beta}$. For comparison, the gray line shows the prediction when $T_{e}$ and $T_{\text {ads }}$ are thermalized. Bottom panel: Integrated desorption rates predicted by the friction model for various activation energies in the $\eta_{\text {adiabatic }}<\eta_{\text {diabatic }} T_{\text {el }}^{\beta}$ case. (b) Adsorbate temperatures predicted by the friction model for two excitation pulses arriving with a delay of $400 \mathrm{fs}$. The dashed line shows the case corresponding to $\eta_{\text {adiabatic }}>\eta_{\text {diabatic }} T_{\mathrm{el}}^{\beta}$, while the dotted line corresponds to $\eta_{\text {adiabatic }}<\eta_{\text {diabatic }} T_{\mathrm{el}}^{\beta}$. For comparison, the gray line shows the electron temperature in graphene.

photoexcitation, electron-phonon scattering leads to cooling of the electron bath and heating of optical phonons. This is followed by relaxation of the hot electron-phonon system on the time scale of a few picoseconds. To properly model the interaction of the adsorbed molecules with the energy absorbed in the graphene sheet one can define a coupling between the three important temperatures in the system; $T_{\mathrm{el}}, T_{\mathrm{ph}}$, and $T_{\text {ads }}$ for the electron, phonon, and adsorbate temperatures, respectively. This description is known as the empirical friction model and has been extensively applied to the study of surface desorption kinetics [26-31]. While the details of calculating the ultrafast excitation and relaxation of $T_{\mathrm{el}}$ and $T_{\mathrm{ph}}$ in graphene have been extensively discussed in the literature [2,3,23,24], we follow the procedure outlined in Ref. [26] to model the coupling to the molecular adsorbates. Briefly, the adsorbate is coupled to the graphene heat baths through a coupling 
constant known as the electronic friction parameter $\eta_{\mathrm{el}}$, related through

$$
\frac{d T_{\mathrm{ads}}}{d t}=\eta_{\mathrm{el}}\left(T_{\mathrm{el}}-T_{\mathrm{ads}}\right) .
$$

Here, the coupling to the phonon thermal bath is assumed to be negligible. There are two justifications for this approach: First, a purely electron mediated response has been observed in the literature for molecular oxygen desorbing from a variety of other conducting interfaces [27-29]. Secondly, the fast relaxation time scales seen in Fig. 4(a) suggest a direct coupling to the fastest measurable relaxation in graphene, which is associated with the electron temperature $[2,23]$. The friction parameter itself is often assumed to be temperature dependent $[26,27,30]$, with the phenomenological form

$$
\eta_{\mathrm{el}}=\eta_{\text {adiabatic }}+\eta_{\text {diabatic }} T_{\mathrm{el}}^{\beta},
$$

where $\beta$ is a constant in the range $2-4$ [27]. The adiabatic term, $\eta_{\text {adiabatic }}$, describes coupling to the adsorbate through many small energy transfer events, while the diabatic term, $\eta_{\text {diabatic }}$, accounts for nonlinearities due to one off, high energy transfer events. Figure 4(b) shows the temperature dependence of the adsorbate bath for different forms of the coupling constant $\eta_{\mathrm{el}}$, depending on whether the adiabatic or diabatic term dominates the dynamics. Since it is difficult to separate the effect of $T^{\beta}$ from $\eta_{\text {diabatic }}$, we simply take $\beta=4$ (as found for oxygen bound to metal surfaces [27]) and vary $\eta_{\text {diabatic }}$, fixing $\eta_{\text {adiabatic }}$ (for simplicity, assuming a value of $7.5 \times 10^{10}$ $\mathrm{s}^{-1}$ ). We find that when $\eta_{\text {adiabatic }}>\eta_{\text {diabatic }} T_{\text {el }}^{\beta}$ (dashed line) the dynamics are very slow, as $T_{\text {ads }}$ is decoupled from $T_{\mathrm{el}}$, and one observes a very slow rise in $T_{\text {ads }}$. When $\eta_{\text {adiabatic }}<\eta_{\text {diabatic }} T_{\mathrm{el}}^{\beta}$ (dotted line) the temporal dynamics are much faster, due to the nonlinear weighting of $T_{\text {ads }}$ to $T_{\mathrm{el}}$ on short time scales, and one observes effects of the individual pulses, labeled pulse 1 and pulse 2 in the figure. The middle panel of Fig. 4(a) shows the time integrated desorption rate corresponding to the adsorbate temperatures shown in Fig. 4(b). The rate of desorption $r(t)$ is calculated through an Arrhenius-type equation

$$
r_{d}(t) \propto e^{-E_{A} /\left[k_{B} T_{\text {ads }}(t)\right]},
$$

where $E_{A}$ is the energy of adsorption (taken for now to be $500 \mathrm{meV}$ ). Two distinct behaviors are seen: For the $\eta_{\text {adiabatic }}>$ $\eta_{\text {diabatic }} T_{\text {el }}^{\beta}$ case (dashed line), we observe an integrated desorption rate that increases with pulse separation. For comparison, we plot the integrated desorption rate expected if $T_{\text {ads }}$ and $T_{e}$ are thermalized [if $\eta_{\text {adiabatic }}$ or $\eta_{\text {diabatic }}$ tend to infinity (gray line)]. Both of these cases predict very different dynamics to those observed in experiment [top panel of Fig. 4(a)]. In contrast, for the $\eta_{\text {adiabatic }}<\eta_{\text {diabatic }} T_{\text {el }}^{\beta}$ case (dotted line), we see a rapid decrease in integrated desorption rate with pulse separation. Again, in this last case, fast dynamics occur due to the nonlinear weighting of $T_{\text {ads }}$ to $T_{\mathrm{el}}$ on short time scales. It is important to note that such behavior could arise for many different combinations of $\eta_{\text {diabatic }}$ and $\beta$. Nevertheless, it is clear that to describe our experimental data we require $\eta_{\text {adiabatic }}<\eta_{\text {diabatic }} T_{\mathrm{el}}^{\beta}$. It is important to note that by varying the size of $\eta_{\text {adiabatic }}$ any integrated desorption curve bounded by the solid gray lines in Fig. 4(b) can be achieved. However only by increasing $\eta_{\text {diabatic }} T_{\mathrm{el}}^{\beta}$ can the highly nonlinear behavior be observed. A similar observation has been made previously for molecular oxygen binding to metal interfaces [27].

The bottom panel of Fig. 4(a) shows the dependence on $E_{A}$. One observes that, on increasing $E_{A}$, the correlation dynamics are faster, due to the exponential dependence of desorption rate on $E_{A}$. It is interesting to note that we require a relatively high $E_{A}$ of many hundreds of meV in order to describe dynamics similar to those measured in our experiment [top panel of Fig. 4(a)], as one requires an activation energy significantly greater than the nonequilibrium electron thermal energies generated in our experiment. Such large binding energies are in distinct contrast to molecular binding energies of oxygen on graphene predicted from DFT calculations, $<60 \mathrm{meV}$ [32]. There are several possible explanations for this large discrepancy. It is possible that the wrinkled nature of real graphene, and the presence of substrate and/or defects could substantially increase molecular binding energies [33]. As suggested by Szymanski et al. [26], there is also an open question as to whether photodesorption energies are entirely comparable to the thermal desorption energies in these kinds of systems. Furthermore, it should be noted that the nature of our methodology will weigh our results towards more strongly bound species, as these will have the largest effect on Fermi level.

In conclusion, we have measured oxygen binding to graphene surfaces using the unique sensitivity of the graphene conductivity to bound surface species. Using two pulse correlation measurements we observe very fast dynamics $(\approx 100 \mathrm{fs})$, which suggests a strong coupling between electronic and adsorbate thermal baths. By comparing our results to an empirical friction model we suggest a diabatically driven desorption mechanism with a rather large energy of desorption of several hundred meV.

We thank P. R. Wilkins for technical assistance with the project and S. J. Green for his valuable comments. This work was funded by the EPSRC (Grants No. EP/K041215/1 and No. EP/G036101/1).
[1] D. Brida, A. Tomadin, C. Manzoni, Y. J. Kim, A. Lombardo, S. Milana, R. R. Nair, K. S. Novoselov, A. C. Ferrari, G. Cerullo, and M. Polini, Nat. Commun. 4, 1987 (2013).

[2] P. J. Hale, S. M. Hornett, J. Moger, D. W. Horsell, and E. Hendry, Phys. Rev. B 83, 121404 (2011).

[3] H. Wang, J. H. Strait, P. A. George, S. Shivaraman, V. B. Shields, M. Chandrashekhar, J. Hwang, F. Rana, M. G. Spencer, C. S. Ruiz-Vargas, and J. Park, Appl. Phys. Lett. 96, 081917 (2010).
[4] B. Y. Sun, Y. Zhou, and M. W. Wu, Phys. Rev. B 85, 125413 (2012).

[5] S. Kaniyankandy, S. Rawalekar, and H. N. Ghosh, J. Phys. Chem. C 116, 16271 (2012).

[6] S.-Z. Liang, G. Chen, A. R. Harutyunyan, M. W. Cole, and J. O. Sofo, Appl. Phys. Lett. 103, 233108 (2013).

[7] M. W. Iqbal, A. K. Singh, M. Z. Iqbal, and J. Eom, J. Phys.: Condens. Matter 24, 335301 (2012). 
[8] W. Yuan and G. Shi, J. Mater. Chem. A 1, 10078 (2013).

[9] F. Schedin, A. Geim, S. Morozov, E. Hill, P. Blake, M. Katsnelson, and K. Novoselov, Nat. Mater. 6, 652 (2007).

[10] E. Hill, A. Vijayaragahvan, and K. Novoselov, IEEE Sens. J. 11, 3161 (2011).

[11] Y. Sato, K. Takai, and T. Enoki, Nano Lett. 11, 3468 (2011).

[12] A. Kaverzin, S. Strawbridge, A. Price, F. Withers, A. Savchenko, and D. Horsell, Carbon 49, 3829 (2011).

[13] A. A. Kaverzin, A. S. Mayorov, A. Shytov, and D. W. Horsell, Phys. Rev. B 85, 075435 (2012).

[14] C. Docherty, C. Lin, H. Joyce, R. Nicholas, L. Herz, L. Li, and M. Johnston, Nat. Commun. 3, 1228 (2012).

[15] S. Ryu, L. Liu, S. Berciaud, Y.-J. Yu, H. Liu, P. Kim, G. W. Flynn, and L. E. Brus, Nano Lett. 10, 4944 (2010).

[16] Y. Yang and R. Murali, Appl. Phys. Lett. 98, 093116 (2011).

[17] H. Liu, S. Ryu, Z. Chen, M. L. Steigerwald, C. Nuckolls, and L. E. Brus, J. Am. Chem. Soc. 131, 17099 (2009).

[18] F.-J. Kao, D. G. Busch, D. Cohen, D. Gomes da Costa, and W. Ho, Phys. Rev. Lett. 71, 2094 (1993).

[19] P. Giannozzi, R. Car, and G. Scoles, J. Chem. Phys. 118 (2003).

[20] We carried out a transfer matrix calculation using the approach outlined in Born and Worf, Principles of Optics, 7th ed. (Cambridge University Press, Cambridge, UK, 1999), pp. 70-74. The refractive indices of the $0.3 \mathrm{~nm}$ graphene (graphite), $300 \mathrm{~nm}$ silicon dioxide, and $1 \mathrm{~mm}$ silicon layers were taken to be $2.7+1.4 i, 1.48$, and $3.7+0.5 i$, taken from E. D. Palik, Handbook of Optical Constants of Solids (Elsevier, New York, 1998).
[21] A. Roberts, D. Cormode, C. Reynolds, T. Newhouse-Illige, B. J. LeRoy, and A. S. Sandhu, Appl. Phys. Lett. 99, 051912 (2011).

[22] M. Currie, J. D. Caldwell, F. J. Bezares, J. Robinson, T. Anderson, H. Chun, and M. Tadjer, Appl. Phys. Lett. 99, 211909 (2011).

[23] C. H. Lui, K. F. Mak, J. Shan, and T. F. Heinz, Phys. Rev. Lett. 105, 127404 (2010).

[24] F. Rana, P. A. George, J. H. Strait, J. Dawlaty, S. Shivaraman, M. Chandrashekhar, and M. G. Spencer, Phys. Rev. B 79, 115447 (2009).

[25] M. Breusing, S. Kuehn, T. Winzer, E. Malić, F. Milde, N. Severin, J. P. Rabe, C. Ropers, A. Knorr, and T. Elsaesser, Phys. Rev. B 83, 153410 (2011).

[26] P. Szymanski, A. L. Harris, and N. Camillone, III, Surf. Sci. 601, 3335 (2007).

[27] P. Szymanski, A. L. Harris, and N. Camillone, J. Chem. Phys. 126, 214709 (2007).

[28] J. A. Misewich, A. Kalamarides, T. F. Heinz, U. Höfer, and M. M. T. Loy, J. Chem. Phys. 100, 736 (1994).

[29] J. Misewich, S. Nakabayashi, P. Weigand, M. Wolf, and T. Heinz, Surf. Sci. 363, 204 (1996).

[30] H. Arnolds, D. A. King, and I. M. Lane, Chem. Phys. 350, 94 (2008).

[31] J. Gladh, T. Hansson, and H. Öström, Surf. Sci. 615, 65 (2013).

[32] M.-T. Nguyen, J. Phys.: Condens. Matter 25, 395301 (2013).

[33] D. W. Boukhvalov and M. I. Katsnelson, J. Phys. Chem. C 113, 14176 (2009). 